\title{
MATERNAL OUTCOMES OF PREGNANCY-RELATED ACUTE KIDNEY INJURY
}

\author{
Muhammad Irfan Khattak, Samina Naseem Khattak, Ayesha Imran, Khawar Kamal, Umairah Yaqoob \\ Combined Military Hospital Kharian/National University of Medical Sciences (NUMS) Pakistan
}

\begin{abstract}
Objective: To determine the frequency of Pregnancy-related acute kidney injury in the Pakistani population and its effect on maternal health.

Study Design: Cross-sectional study.

Place and Duration of the Study: Department of Nephrology and Obstetrics and Gynecology, Combined Military Hospital Kharian, from Jan 2018 to Dec 2020.

Methodology: All pregnant women with normal renal functions at the time of enrolment in the study and no previous history of any kidney disease or renal procedure in the recent past (at least 3 months) before conception were included in our study at Combined Military Hospital Kharian. All participants had serum creatinine tested at enrolment and were inquired specifically about any history of renal disease or any renal procedure in the recent past (at least 3 months old).

Results: Mean maternal age in the study was $29 \pm 7.67$ years however it ranged from 16-41 years. Around a quarter of acute kidney injury cases occurred in the first and second trimesters combined, with $26(12 \%)$ and $24(11 \%)$ cases in the first and second trimesters, respectively. Thirty percent of acute kidney injury cases were diagnosed in the third trimester. A substantial number $58(27 \%)$ of cases developed azotemia which persisted beyond three months and developed chronic kidney disease of various stages. Regarding maternal outcomes, $166(77 \%)$ mothers recovered completely. However, $43(20 \%)$ patients had some degree pf azotemia, a few cases required permanent renal replacement therapy.

Conclusion: Acute kidney injury is a common problem in our community leading to suboptimal maternal outcomes. Overall prognosis is relatively better but there is a definitive association with the development of chronic kidney disease and even permanent dialysis dependency.
\end{abstract}

Keywords: Acute kidney injury, Hemolytic uraemic syndrome thrombotic thrombocytopenic purpure (TTP), Renal failure in pregnancy.

How to Cite This Article: Khattak I, Khattak SN, Imran A, Kamal K, Yaqoob U. Maternal Outcomes of Pregnancy-Related Acute Kidney Injury. Pak Armed Forces Med J 2021; 71(6): 2057-2060.

Doi: https://doi.org/10.51253/pafmi.v6i6.5813

This is an Open Access article distributed under the terms of the Creative Commons Attribution License (https://creativecommons.org/licenses/by-nc/4.0/), which permits
unrestricted use, distribution, and reproduction in any medium provided the original work is properly cited.

\section{INTRODUCTION}

Acute kidney injury represents a challenging clinical problem, especially when it occurs at some stage during pregnancy for both obstetricians and nephrologists. Pregnancy-related acute kidney injury has major implications for both maternal and fetal health and pregnancy outcomes. ${ }^{1}$ Despite recent advances in obstetrics and nephrology care overall pregnancy-related kidney injury remains unchartered territory. Causes that are responsible for AKI in the general public can cause AKI during pregnancy, but Pr-AKI may have causes specifically related to pregnancy. The true incidence of AKI is not known exactly because of lack of consensus on the definition of AKI in pregnancy as hyperfiltration during pregnancy leads to subnormal values of urea and creatinine so what appears normal in ordinary population may represent the extremely compromised renal function and is likely to be missed

Correspondence: Dr Umairah Yaqoob, Department of Gynaecology, Pak Emirates Military Hospital, Rawalpindi Pakistan

Received: 08 Dec 2020; revision received: 25 Oct 2021; accepted: 29 Oct 2021 by treating physicians. ${ }^{2}$ While the freq-uency of Pr-AKI requiring dialysis in developed coun-tries is low ( 1 in 2000 cases), in underdeveloped count-ries incidence of up to $10-20 \%$ has been reported which is a major health dilemma. ${ }^{3}$

There is a dire need to explore this issue especially in underdeveloped world countries and get some valuable data and insight into this clinical conundrum. The information obtained will enable government agencies and health professionals to better plan and treat this potentially fatal health issue.

\section{METHODOLOGY}

This was a cross-sectional study was carried from January 2018 to December 2020. Previously a Chinese study by Diankun et al. has reported, "AKI occurred in $393(3.6 \%)$ and $402(3.7 \%)$ of the pregnant women, respectively, giving rise to an overall AKI incidence of $7.3 \%$. While hospital coding would have identified less than 5\% of AKI episodes." Moreover, he also mentioned that, "incidence of AKI during pregnancy in developing countries, such as India, and Pakistan ranged 
from $0.02-11.5 \% "$. Ali et al. mentioned obstetric related AKI incidence of $9-11 \%$ in 2007. The sample size was calculated using the Raosoft sample size calc-ulator. With a 5\% margin of error and $95 \%$ confidence interval estimated size was around 259 cases due to time and financial constraints we swapped to non-probability consecutive sampling.

Inclusion Criteria: All the ladies reporting for the antenatal visit were examined and evaluated for evidence of AKI and those who fulfilled our criteria of AKI were included in the study.

Exclusion Criteria: Patients suffering from chronic kidney disease, hypertension, diabetes mellitus, renal transplantation, liver failure, heart failure or any malignancy were excluded. Those patients who had undergone some urological procedure in the preceding three months were also excluded.

AKI was diagnosed and staged by Kidney Disease Improving Global Outcomes (KDIGO) criteria. Informed consent was taken. Approval of the Ethical committee was taken Ref: no. 1100/adm/2. The primary outcomes were the Frequency proportion of PrAKI. Secondary outcomes were in-hospital and postdischarge maternal outcomes including mortality, the requirement for dialysis, renal recovery, and length of stay in hospital. Full clinical examination was done by consultants or registrars. A blood sample was collected in a 10cc sterile syringe and sent to the hospital laboratory by hospital staff. Complete blood count, blood urea, serum creatinine, ESR, CRP and urine analysis) were done. Renal biopsy was performed on patients who had no clearly defined cause for their renal insufficiency for more than 3 weeks.

Data was entered in Statistical Package for the social sciences (SPSS) version 23. Descriptive statistics were calculated as means, standard deviation and percentages for Age, maternal outcome, serum creatinine, survival, and long-term morbidities. The significance of the difference in means was calculated using paired sample t-test, the $p$-value of $\leq 0.05$ was labelled as significant.

\section{RESULTS}

A total of 216 population developed AKI during our study time period and were included in the study. The mean age of the mother with Pr-AKI was $29 \pm 7.67$ years. We divided mothers into three main age groups. Pr- AKI was not very common in young mothers as 43 $(20 \%)$ mothers with Pr-AKI were in the age group up to 20 years. Similar figures $43(23 \%)$ were observed in the age group (21-30) years. An alarming rise was noted in mothers belonging to the $3^{\text {rd }}$ group ( $>30$ years) as $124(57.4 \%)$ belonged to this age group. Therefore, most of the Pr-AKI cases occurred in mothers of advanced age. Regarding maternal outcomes, the overall prognosis was good as 115 (53\%) recovered completely, evidenced by complete clinical and laboratory parameters returning to normal. At the same time, $28(27 \%)$ cases developed azotemia which persisted beyond three months, following the initial AKI event. They were labelled as chronic kidney disease (CKD). A substantial number of cases $9(13.4 \%)$ developed severe enough azotemia to require dialysis. 24 (11\%) mothers belonged to Upper Class in comparison to 72 (33\%) from the middle class shown in Table-I.

Table-I: Demographics and outcomes of the study population.

\begin{tabular}{l|c}
\hline \multicolumn{1}{l|}{ Study Variables } & $\mathbf{n}(\mathbf{0})$ \\
\hline Age & $43(19.9)$ \\
\hline$<20$ years & $49(22.7)$ \\
\hline $21-30$ years & $124(57.4)$ \\
\hline Outcome years & \\
\hline Complete Recovery & $115(53.2 \%)$ \\
\hline Chronic Kidney disease & $58(26.9 \%)$ \\
\hline Dialysis Dependent & $29(13.4 \%)$ \\
\hline Death & $14(6.5 \%)$ \\
\hline Socio Economic Class & $24(11.1 \%)$ \\
\hline Upper Class & $72(33.3 \%)$ \\
\hline Middle Class & $120(55.6 \%)$ \\
\hline Lower Class &
\end{tabular}

Most of the Pr-AKI was observed in lowerclass $120(55 \%)$, majority of women with AKI belonged to rural areas $121(56 \%)$ which were more than Urban areas $96(44.4 \%)$. We divided timings of occurrence of AKI into four-time frames i.e. The three trimesters and the post-partum period. Around a quarter of acute kidney, injury cases occurred in the first and second trimesters combined, with $26(12 \%)$ and $24(11 \%)$ cases of acute kidney injury occurring in the first and second trimester, respectively. Thirty percent of acute kidney injury cases were diagnosed in the third trimester. A substantial number $58(27 \%)$ of cases developed azotemia which persisted beyond three months and developed chronic kidney disease of various stages. Regarding maternal outcomes, 166 (77\%) mothers recovered completely. However, 43 (20\%) patients had some degree pf azotemia, a few cases required permanent renal replacement therapy. Regarding causes of AKI, sepsis and postpartum haemorrhage were the commonest causes of AKI accounting for $>60 \%$ of cases. For further details of other causes are shown in the Figure. 


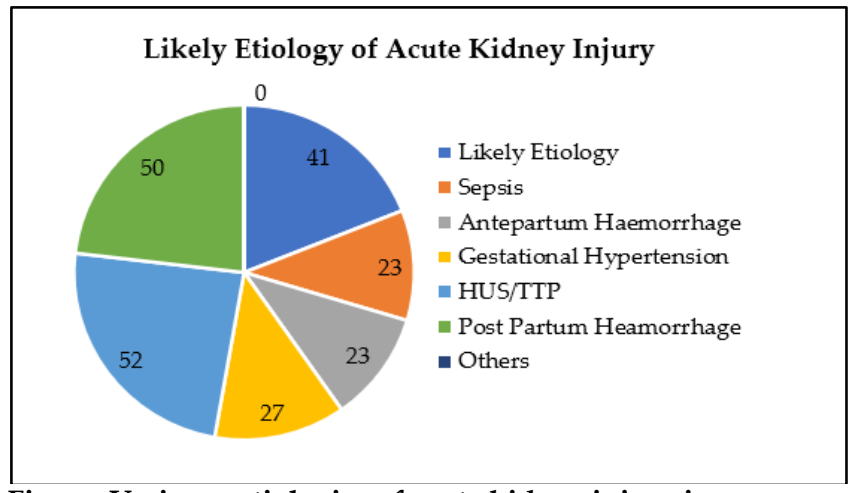

Figure: Various aetiologies of acute kidney injury in pregnancy.

The mean creatinine varied widely between the three trimesters. It reduced to the lowest value of $186 \pm$ $92.11 \mathrm{umol} / 1$ in the second trimester after starting at the mean value of $203 \pm 249,5 \mathrm{umol} / 1$ in the first trimester. There was no statistically significant difference between both trimesters $p=0.236$ the highest mean creatinine was found in the third trimester at $310 \pm 283.35$ umol/1, there was a statistically significant difference between the second and third-trimester $p<0.001$ and first and third-trimester $p<0.001$ shown in Table-II.

Table-II: Comparison of serum creatinine and trimester.

\begin{tabular}{l|c|c|c}
\hline Parameters & \multicolumn{2}{|c|}{ Trimester } & $p$-value \\
\hline \multirow{4}{*}{$\begin{array}{l}\text { Serum } \\
\text { creatinine } \\
\text { (umol/1) }\end{array}$} & 1st Trimester & 2nd Trimester & 0.236 \\
\cline { 2 - 3 } & $203 \pm 249$ & $186 \pm 92.106$ & \\
\cline { 2 - 3 } & 2nd Trimester & 3rd Trimester & $<0.001$ \\
\cline { 2 - 3 } & $186 \pm 92.106$ & $310 \pm 283$ & \\
\cline { 2 - 3 } & 3rd Trimester & 1st Trimester & $<0.001$ \\
\cline { 2 - 3 } & $310 \pm 283$ & $203 \pm 249$ & \\
\hline
\end{tabular}

\section{DISCUSSION}

Obstetrics related AKI is a huge health-related problem faced by both developing and underdeveloped countries. ${ }^{6}$ However recently a decline has been noted in the incidence of Pr-Aki in developed countries contrary to underdeveloped countries. A study carried out in Italy showed that the frequency of obstetrics related AKI has reduced from a total incidence of $43 \%$ to $0.5 \%$ of total reported cases of AKI and $1 / 3000$ to $1 / 18,000$ of total pregnancies in three decades. ${ }^{7}$

The above-mentioned trend is causing increasing problems for developing countries as their health resources are limited and renal failure is a resourcedraining disease with high morbidity and mortality. Even rich and technologically advanced countries like Australia are struggling to fight this disease. ${ }^{8}$ There are a few local studies to give an insight into this complex issue. A recent local study was carried out by Haroon et al. They found that the mean age was 29.90 years. Out of 111 cases, 10 (9\%) developed AKI in the 1st tri- mester, $12(10.8 \%)$ in the 2 nd trimester, $13(11.7 \%)$ cases in the 3rd trimester, and major bulk of the cases of AKI occurred in the post-partum period $=76(68.4 \%)$. The causes of Pr-AKI were multiple and varied in different patients. The frequencies of complications leading to AKI were observed individually. ${ }^{9}$ Similarly maximum cases of AKI occurred in the post-partum period. However, their percentage $(68 \%)$ was well more than ours at $47 \%$. In addition, they studied the causes of AKI individually, but it was out of scope in our study. Their study did not show any association of AKI with advanced maternal age but in our study frequency of AKI was highest in the age group (30 and above) mothers as $(57 \%)$ belonged to this age group.

Rizwan et al, at Karachi, described meaning age 26 (29 years in our study). They related AKI to gravidity also which was not the case in our study. Regarding maternal outcome, they quoted $68 \%$ of a mother with AKI underwent dialysis which is remarkably high compared to ours 29 (13\%). In their study three-month follow-up showed complete resolution of AKI in $14(34.2 \%)$ patients, partial resolution in 7 (17\%), endstage renal disease in $10(24.4 \%)$, and death in 10 $(24.4 \%)$ patients. ${ }^{10}$ In comparison, our population 115 (53\%) recovered completely, 58 (27\%) developed CKD and $29(13 \%)$ became permanently dialysis-dependent. Death frequency was exceptionally low in our study at $14(7 \%)$ compared to them. So clearly our maternal outcomes were better than their population and the difference may have been due to diverse cultural backgrounds, economic classes, and varying health resource access facilities. ${ }^{10}$

To add to the confusion there is wide variation in maternal outcome figures in numerous studies carried out in different counties and various setups within the same country. For example, Elfeky et al had far better maternal outcomes compared to ours and other studies in South Asia. They reported complete recovery in $87 \%$ of the patients, which is more than all the studies quoted above. Similarly, only $4 \%$ of mothers ended up in dialysis and only $1.4 \%$ died during the study period. These differences might have been due to distinct cultural and medical facilities in Egypt, but their study population was only 70 patients which are relatively insignificant compared to our study $>200$ patients. Furthermore, we followed up patients for three months after the event of AKI and assigned them to various outcomes. Their follow-up time was 6 months which may have given more time for kidneys to recover completely and hence remarkably high rates of comp- 
lete recovery in their population. ${ }^{1}$ Another study from Malawi by Cook was a landmark study on the same issue. They studied an exceptionally large population of 322 patients with Pr-AKI and the incidence of AKI was $8 \%$. Surprisingly enough in contradiction to most of the world literature, no women with AKI died or required dialysis and complete renal recovery occurred in $22(84.6 \%)$ cases. These results were most promising in all the studies compared so far, ${ }^{11}$ Many of their results were not in keeping with most of the published literature as their results were better than most of the published literature..$^{5,12-18}$

Therefore, in nutshell, our findings were in keeping with most of the local studies and literature in under-developed countries. However, outcome figures varied widely in different studies which may be the result of different study designs, population sizes and variable definitions of AKI. ${ }^{19-24}$ All literature of the world is unanimous on the fact that high morbidity and mortality is associated with Pr-AKI and this issue needs early recognition and prompt management.

\section{CONCLUSION}

Acute kidney injury is a common problem complicating pregnancies in district Gujrat of Pakistan. However, the overall prognosis is good as most of the mothers developing Aki during pregnancy regained normal renal function. Despite all this, a limited proportion of patients ended up in potentially disabling and life-threatening complications of chronic kidney disease.

\section{Conflict of Interest: None.}

\section{Authors' Contribution}

MIK: Direct contribution, SNK: Direct contribution, AI: Intellectual contribution, KK: Intellectual contribution, UY: Intellectual contribution.

\section{REFERENCES}

1. Elfeky H, Mohammed R, Ahmed T, Ibrahim A, Abo El, Makarem I. Pregnancy related acute kidney injury. Nephrol Dial Transplant 2018; 33(suppl-1): i413-i413.

2. Krane NK. Acute renal failure in pregnancy. Arch Intern Med 1988; 148(11): 2347-2357.

3. Nwoko R, Plecas D, Garovic VD. Acute kidney injury in the pregnant patient. Clin Nephrol 2012; 78(6): 478-486.

4. Najar MS, Shah AR, Wani IA, Reshi AR, Banday KA, Bhat MA, et al. Pregnancy-related acute kidney injury: A single-centre experience from the Kashmir Valley. Indian J Nephrol. 2008 18(4): 159161.

5. Prakash J, Ganiger VC, Prakash S, Iqbal M, Kar DP, Singh U, et al. Acute kidney injury in pregnancy with special reference to pregnancy-specific disorders: a hospital-based study (2014-2016). J Nephrol 2018; 31(1): 79-85.

6. Acute kidney injury related to pregnancy in developing countries: aetiology and risk factors in an intensive care unit.-PubMedNCBI [Internet]. Available at: https://www.ncbi.nlm.nih.gov/ pubmed/22144377[Accessed on Aug 28, 2019].
7. Stratta P, Besso L, Canavese C, Grill A, Todros T, Benedetto C, et al. Is Pregnancy-Related Acute Renal Failure a Disappearing Clinical Entity?. Ren Fail 1996; 18(4): 575-584.

8. Safi N, Sullivan E, Li Z, Brown M, Hague W, McDonald S, et al. Serious kidney disease in pregnancy: an Australian national cohort study protocol. BMC Nephrol 2019; 20(1): 230-233.

9. Haroon F, Dhrolia MF, Qureshi R, Imtiaz S, Ahmed A. Frequency of pregnancy-related complications causing acute kidney injury in pregnant patients at a tertiary care hospital. Saudi J Kidney Dis Transplant 2019; 30(1): 194-201.

10. Bokhari SRA, Inayat F, Jabeen M, Sardar Z, Saeed S, Malik AM, et al. Characteristics and outcome of obstetric acute kidney injury in pakistan: a single-center prospective observational study. Cureus 2018; 10(9): e3362.

11. Cooke WR, Hemmilä UK, Craik AL, Mandula CJ, Mvula P, Msusa A, et al. Incidence, aetiology, and outcomes of obstetricrelated acute kidney injury in Malawi: a prospective observational study. BMC Nephrol 2018; 19(1): 25-28.

12. Liu D, He W, Li Y, Xiong M, Wang L, Huang J, et al. Epidemiology of acute kidney injury in hospitalized pregnant women in China. BMC Nephrol 2019; 20(1): 67-70.

13. Ye W, Shu H, Yu Y, Li H, Chen L, Liu J, et al. Acute kidney injury in patients with HELLP syndrome. Int Urol Nephrol 2019; 51(7): 1199-1206.

14. Hall DR, Conti-Ramsden F. Acute kidney injury in pregnancy including renal disease diagnosed in pregnancy. Best Pract Res Clin Obstet Gynaecol 2019; 57(1): 47-59.

15. Gopalakrishnan N, Dhanapriya J, Muthukumar P, Sakthirajan R, Dineshkumar T, Thirumurugan S, et al. Acute kidney injury in pregnancy-a single-centre experience. Ren Fail 2015; 37(9): 14761480.

16. Piccoli GB, Zakharova E, Attini R, Ibarra Hernandez M, Covella $\mathrm{B}$, Alrukhaimi $\mathrm{M}$, et al. Acute kidney injury in pregnancy: the need for higher awareness. a pragmatic review focused on what could be improved in the prevention and care of pregnancyrelated AKI, in the Year Dedicated to Women and Kidney Diseases. J Clin Med 2018; 7(2): 18-20.

17. Ganesan C, Maynard SE. Acute kidney injury in pregnancy: the thrombotic microangiopathies. J Nephrol 2011; 24(5): 554-563.

18. Kozlovskaya NL, Korotchaeva YV, Shifman EM. Atypical hemolytic-uremic syndrome is one of the causes of acute kidney injury in pregnant women. Ter Arkh 2018; 90(6): 28-34.

19. Trakulsrichai S, Paisanrodjanarat B, Sriapha C, Tongpoo A, Udomsubpayakul U, Wananukul W. Clinical outcome of paraquat poisoning during pregnancy. Clin Toxicol 2019; 57(8): 712-717.

20. Meibody F, Jamme M, Tsatsaris V, Provot F, Lambert J, Frémeaux-Bacchi V, et al. Post-partum acute kidney injury: sorting placental and non-placental thrombotic microangiopathies using the trajectory of biomarkers. Nephrol Dial Transplant 2020; 35(9): 1538-1546.

21. Ramachandran R, Nayak S, Anakutti HP, Yadav AK, Nada R, Jain V, et al. Postpartum renal cortical necrosis is associated with atypical hemolytic uremic syndrome in developing countries. Kidney Int Rep 2019; 4(3): 420-424.

22. Liu Y, Ma X, Zheng J, Liu X, Yan T. Pregnancy outcomes in patients with acute kidney injury during pregnancy: a systematic review and meta-analysis. BMC Preg Childbirth 2017; 17(1): 235238.

23. Liu D, He W, Li Y, Xiong M, Wang L, Huang J, et al. Epidemiology of acute kidney injury in hospitalized pregnant women in China. BMC Nephrol 2019; 20(1): 67-70.

24. Ali A, Ali MA, Ali MU, Mohammad S. Hospital outcomes of obstetrical-related acute renal failure in a tertiary care teaching hospital. Ren Fail 2011; 33(3): 285-290. 